\title{
Human placenta mesenchymal stem cells suppress airway inflammation in asthmatic rats by modulating Notch signaling
}

\author{
YUANYUAN LI ${ }^{1 *}$, TIANTIAN QU ${ }^{1,2^{*}}$, LIJUN TIAN $^{1}$, TINGTING HAN ${ }^{3}$, YONGJUN JIN $^{2}$ and YUESI WANG ${ }^{1}$ \\ ${ }^{1}$ Medicine and Pharmacy Research Center; ${ }^{2}$ Department of Endocrine Metabolism, Binzhou Medical University, Yantai, \\ Shandong 264003; ${ }^{3}$ Department of Respiratory, Binzhou Medical University Hospital, Binzhou, Shandong 256603, P.R. China
}

Received March 6, 2017; Accepted November 2, 2017

DOI: $10.3892 / \mathrm{mmr} .2018 .8462$

\begin{abstract}
Neurogenic locus notch homolog protein (Notch) signaling mediates intracellular communication and may regulate cell fate decisions, including cell proliferation, differentiation, and apoptosis. Mesenchymal stem cells (MSCs) possess immunomodulatory properties and the potential for use in stem cell replacement treatments. The aim of the present study was to evaluate the therapeutic effects of human placenta-deviated MSCs (hPMSCs) in asthma and to investigate the mechanisms of Notch signaling mediated by transplanted MSCs. A Sprague-Dawley rat ovalbumin (OVA)-sensitized acute asthma model was established and challenged. MSCs derived from human placenta (hPMSCs) were transplanted into the asthmatic rats. Transplantation resulted in reduced Notch-1, Notch-2 and jagged-1, and increased Notch-3, Notch-4 and delta-like ligand (delta)-4 expression in lung, blood, and lymph samples. Notch-1, Notch-2, and jagged-1 expression in OVA-treated rats was significantly decreased compared with controls and
\end{abstract}

Correspondence to: Professor Yongjun Jin, Department of Endocrine Metabolism, Binzhou Medical University, 346 Guanhai Road, Yantai, Shandong 264003, P.R. China

E-mail: endojin@126.com

Professor Yuesi Wang, Medicine and Pharmacy Research Center, Binzhou Medical University, 346 Guanhai Road, Yantai, Shandong 264003, P.R. China

E-mail: wys7416@163.com

${ }^{*}$ Contributed equally

Abbreviations: MSCs, mesenchymal stem cells; hPMSCs, human placenta mesenchymal stem cells; Treg, regulatory $\mathrm{T}$ cell; Th, helper T cell; BM-MSCs, bone marrow-derived mesenchymal stem cells; AHR, airway hyperreactivity; BALF, bronchoalveolar lavage fluid; NS, normal saline; RT-qPCR, reverse transcription-quantitative polymerase chain reaction; Notch, neurogenic locus notch homolog protein; OVA, ovalbumin; delta, delta-like ligand; IFN- $\gamma$, interferon- $\gamma$; Ig, immunoglobulin; IL, interleukin; DCs, dendritic cells; FoxP3, forkhead box protein P3; BCA, bicinchoninic acid

Key words: asthma, hPMSCs, Notch, lymph, rats
hPMSC-treated rats; however, Notch-3, Notch-4 and delta-4 expression was significantly increased. Serum interferon- $\gamma$ significantly increased after hPMSCs transplantation, whereas interleukin-4 and immunoglobulin E decreased. In OVA-treated rats, Notch-1, Notch-2 and jagged-1 levels were increased in the lymph compared with the blood, although Notch-4 and delta-4 levels were decreased. Peribronchial infiltration of cells and goblet cell hyperplasia were markedly decreased in the OVA + hPMSCs group compared with those in the OVA-treated and control groups. Alterations in Notch signaling pathway expression were accompanied by decreased inflammatory cell infiltration, goblet cell hyperplasia and mucus production in lung tissues. The results of the present study are consistent with hPMSC suppression of asthma symptoms and inflammation by regulating the Notch signaling pathway in the rat asthma model.

\section{Introduction}

Asthma symptoms frequently include airway obstruction, airway hyperreactivity (AHR), and airway inflammation. Allergic asthma is a chronic inflammatory dysfunction exacerbated by eosinophilia and T-helper type 2 (Th2) cellactivation. Traditional asthma therapies, including corticosteroids and antihistamines, have limitations and side effects $(1,2)$. Persistent inflammation does not reverse chronic airway remodeling and can lead to tissue damage. Neurogenic locus notch homolog protein (Notch) signaling is a well-conserved pathway involved in the development of immune cells, and dendritic cells (DCs) and T cells express Notch receptors and ligands. Notch signaling is involved in T-cell activation and proliferation, as reported by Rutz et al (3), who demonstrated that delta-like ligand (delta)-1 and jagged-1 inhibited T-cell activation, whereas delta-4 promoted T-cell proliferation. In mice and humans, differentiation of forkhead box protein P3 (Fox P3)-positive regulatory $\mathrm{T}$ cells (Tregs) from naive $\mathrm{CD} 4^{+} \mathrm{T}$ cells followed transforming growth factor- $\beta 1$ signal activation of the Notch pathway $(4,5)$. Mesenchymal stem cells (MSCs) are self-renewing and multipotential $(6,7)$, and due to their unique immunological characteristics, including immunoregulatory activity and low immunogenicity, MSCs are attractive tools for regenerative medicine $(8,9)$. MSCs may regulate T-cell proliferation, inhibit B-cell proliferation $(10,11)$ and affect the regulation of immune responses 
by DCs (12) by inducing Treg formation from naive $\mathrm{CD} 4^{+} \mathrm{T}$ cells $(13,14)$. MSCs are able to expand anti-specific Tregs to prevent autoimmunity $(15,16)$ and have been demonstrated to directly or indirectly induce Tregs in the regulation of DCs. MSC-induced Tregs have an important immunosuppressive effect on allergic asthma (17). The involvement of the Notch pathway in the MSC modulation of the adaptive immune response in asthma is not completely understood. The objective of the present study was to investigate the importance of placenta-derived MSCs (hPMSCs) in the modulation of the Notch signaling pathway in asthma and to increase understanding of the crosstalk between Notch signaling and MSC modulation of adaptive immune responses. MSCs can regulate the immune system through cytokine production and the Notch pathway, leading to reduced inflammation. Thus, MSC-based therapies may be effective in the treatment of asthma.

\section{Materials and methods}

Animal model. A total of 60 Wistar male rats $(185 \pm 12.5 \mathrm{~g})$ were obtained at 6-8 weeks of age from the Shandong Lukang Record Pharmaceutical Co., Ltd. (Jining, China) and were maintained in a specific pathogen-free facility with a $12 \mathrm{~h}$ light/dark cycle at the Medicine and Pharmacy Research Center of Binzhou Medical University (Yantai, China). Rats were housed at $18-26^{\circ} \mathrm{C}$ and $40-70 \%$ relative humidity with free access to food and water. All animal experimentation and procedures were approved by the Institutional Animal Care and Use Committee of Binzhou Medical University.

The asthma rat model was established as previously described (18). Rats were sensitized by intraperitoneal injection of sterile ovalbumin (OVA; $1 \mathrm{mg}$ in $1 \mathrm{ml}$ saline; Sigma-Aldrich; Merck KGaA, Darmstadt, Germany) with $200 \mathrm{mg}$ aluminum hydroxide as an adjuvant, on days 1 and 8 . Following sensitization, the rats were nebulized daily with $1 \%$ OVA (w/v) solution for $30 \mathrm{~min}$ in a $30 \times 24 \times 50-\mathrm{cm}$ chamber between days 15 and 21 . A control group received intraperitoneal injections of saline on days 1 and 8 , and was exposed to aerosolized saline on the provocation days. Rats in the OVA + hPMSC group received $1 \times 10^{6} \mathrm{hPMSCs} / \mathrm{kg}$ bodyweight suspended in $1 \mathrm{ml}$ sterile Dulbecco's PBS via tail vein injections, prior to the final OVA challenge. Rats were sacrificed via cervical dislocation $24 \mathrm{~h}$ subsequent to treatment. The protocol is illustrated in Fig. 1.

Isolation and culture of hPMSCs. hPMSCs were derived from full-term placentas subsequent obtaining informed consent from the donors, with the approval of the Institutional Ethics Committee. The cells were cultured in L-Dulbecco's modified Eagle's medium (Hyclone; GE Healthcare Life Sciences, Logan, UT, USA), with $10 \%$ fetal bovine serum (Hyclone; GE Healthcare Life Sciences) and $100 \mathrm{U} / \mathrm{ml}$ each of penicillin and streptomycin. Cells were cultured at $37^{\circ} \mathrm{C}$ in a humidified atmosphere with $5 \% \mathrm{CO}_{2}$. The immunophenotype of hPMSCs [cluster of differentiation (CD73, CD90, CD105, CD45 and human leukocyte antigen-antigen $\mathrm{D}$ related) were determined by flow cytometry at the third culture passage. Neurogenic, osteogenic, and adipose differentiation were promoted to identify pluripotent hPMSCs.
Lung histology. Rats were anaesthetized with an intraperitoneal injection of $4 \%$ chloral hydrate $(300-350 \mathrm{mg} / \mathrm{kg}$ ) and sacrificed via cervical dislocation; lungs were harvested, perfused with PBS, and fixed in $10 \%$ neutral buffered formalin overnight. Lung tissue was embedded for light microscopy (x200) for the evaluation of inflammatory cell infiltration. Bronchoalveolar lavage fluid (BALF) was collected by cannulation of the trachea and flushing of the left lung bronchus three times with normal saline. The supernatant was discarded following centrifugation at $500 \mathrm{x} \mathrm{g}$ for $10 \mathrm{~min}$ at $25^{\circ} \mathrm{C}$. The total cell, eosinophil, lymphocyte, macrophage and neutrophil counts were obtained following Wright's staining for $\sim 1-2$ min at room temperature. All the obtained cells were counted by a pathological image analysis system (Image-Pro Plus 6.0; Media Cybernetics, Inc., Rockville, MD, USA).

ELISA analysis. Interleukin (IL)-4 (cat. no. R4000), interferon (IFN) $-\gamma$ (cat. no. DY585) and immunoglobulin (Ig)E (cat. no. DY6900) levels were assayed using commercially available ELISA kits purchased from R\&D Systems, Inc. (Minneapolis, MN, USA).

Reverse transcription-quantitative polymerase chain reaction (RT-qPCR) analysis. Total RNA was extracted from lung samples and MNCs using TRIzol reagent (Thermo Fisher Scientific, Inc.), dissolved in diethyl pyrocarbonate in double distilled water and stored at $-80^{\circ} \mathrm{C}$. cDNA was synthesized using a Revert Aid First-Strand cDNA Synthesis kit (Thermo Fisher Scientific, Inc.) and subsequently amplified. qPCR was performed using the TransStart Tip Green qPCR SuperMix (TransGen Biotech Co., Ltd., Beijing, China) on the Real-Time PCR Detection system (iQ5; Bio-Rad Laboratories, Inc., Hercules, CA, USA). The PCR conditions were DNA denaturation at $94^{\circ} \mathrm{C}$ for $30 \mathrm{sec}$, followed by 40 cycles at $94^{\circ} \mathrm{C}$ for $5 \mathrm{sec}, 60^{\circ} \mathrm{C}$ for $30 \mathrm{sec}$ and $72^{\circ} \mathrm{C}$ for $1 \mathrm{~min}$. All experiments were performed according to the manufacturer's protocol. The gene-specific primers were as follows: Notch-1 forward, AAC TCACCAGAGCAGCCTACA; Notch-1 reverse, CCACAT TCCAGCACACTCAA; Notch-2 forward, TGGAGGCAG GAGGAAAGAC; Notch-2 reverse, GATGGGCAAAGGTCA GTAGG; Notch-3 forward, CGGCTTGATTTCCCATACC; Notch-3 reverse, ACACCCAGGACGAAGATGAC; jagged-1 forward, AGTAAACGGGATGGGAACAG; jagged-1 reverse, CAGCAGAGGAACCAGGAAAT; delta-4 forward, GCC CAGACTCCATCCTTACA; delta-4 reverse, GCTCCTGCT AAATGCCAGAC; and GAPDH forward, ACAGCAACA GGGTGGTGGAC; GAPDH reverse, TTTGAGGGTGCA GCGAACTT. PCR results were quantified using the $2^{-\Delta \Delta C q}$ method (19).

Western blot analysis. Lung tissue was homogenized and lysed with radioimmunoprecipitation assay buffer (Beyotime Institute of Biotechnology, Haimen, China) and the supernatant was collected following centrifugation at 12,000 $\mathrm{x}$ g for $5 \mathrm{~min}$ at $4^{\circ} \mathrm{C}$. Protein was quantified using a bicinchoninic acid (BCA) assay with the Pierce BCA Protein Assay kit (Thermo Fisher Scientific, Inc.), according to the manufacturer's protocol; $50 \mu \mathrm{g}$ aliquots of protein were loaded and electrophoresed on a $10 \%$ SDS-PAGE gel, and transferred onto polyvinylidene fluoride membranes. Following blocking 


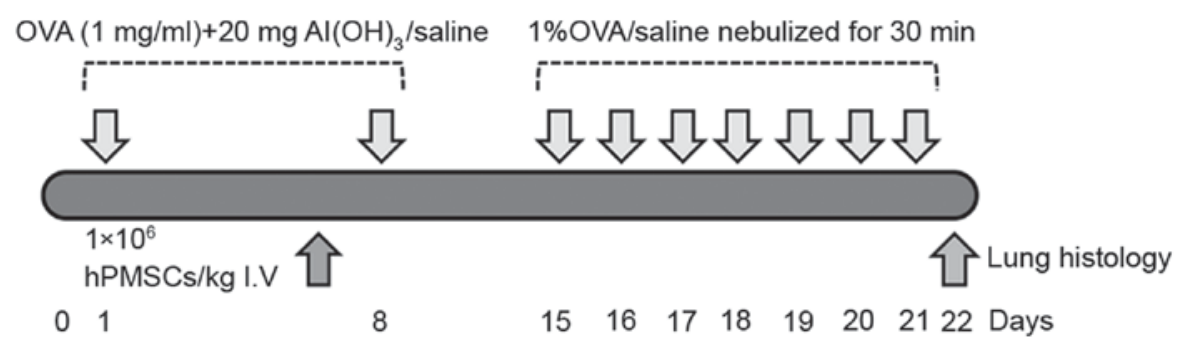

Figure 1. Experimental protocol and groupings. OVA, ovalbumin; hPMSCs, human placenta mesenchymal stem cells; IV, intravenous.

for $2 \mathrm{~h}$ in $5 \%$ non-fat milk at room temperature, membranes were incubated with primary antibodies overnight at $4^{\circ} \mathrm{C}$, rinsed, and incubated for $1 \mathrm{~h}$ with the corresponding secondary antibodies at room temperature for $1 \mathrm{~h}$. Blots were visualized with diaminobenzidine and read with a Clinx GenoSens1600 electrophoresis gel imaging analyzer (Clinx Science Instruments Co., Ltd., Shanghai, China). The bands were semi-quantified using ImageJ 1.51 software (National Institutes of Health, Bethesda, MD, USA). Primary antibodies included anti-Notch-1 (1:500; cat. no. 3447; Cell Signaling Technology, Inc., Danvers, MA, USA), Notch-2 (1:50; 5732; Cell Signaling Technology, Inc.), Notch-3 (1:500; 2889; Cell Signaling Technology, Inc.), jagged-1 (1:50; ab7771; Abcam, Cambridge, UK) and delta-4 (1:500; ab183523; Abcam). Horseradish peroxidase-conjugated secondary antibodies were obtained from Wuhan Sanying Biotechnology (1:500; SA00001-15; Wuhan, China).

Statistical analysis. Statistical calculations were performed using SPSS 18.0 (SSPS, Inc., Chicago, IL, USA). Results are presented as the mean \pm standard deviation. When applicable, one-way analysis of variance and Holm-Sidak tests were used to determine significance. $\mathrm{P}<0.05$ was considered to indicate a statistically significant difference.

\section{Results}

hPMSCs engraftment results in a significant reduction in inflammatory cells in BALF and lung tissue. The cells in the BALF collected from controls, OVA- and hPMSC-treated rats were counted on a hemocytometer. The total number of cells and the number of eosinophils were both significantly greater in the OVA group compared with the OVA + hPMSCs and control groups; these counts were also greater in the OVA + hPMSCs group in the OVA group (Fig. 2). Histological evaluation revealed inflammatory alterations in the bronchial tissue of rats in the OVA group, including inflammatory cell infiltration of the trachea, and surrounding perivascular tissue and subepithelial smooth muscle, along with luminal narrowing. The asthma model was thus successfully established. The inflammatory alterations were significantly reduced in the OVA + hPMSCs group compared with the OVA group. Inflammatory cell infiltration and goblet cell hyperplasia were reduced, mucus secretion was downregulated, and airway smooth muscle proliferation was attenuated. Only slight luminal narrowing was apparent, and only occasional alveolar inflammatory cells were observed (Fig. 3). hPMSCs were thus demonstrated to alleviate the development of asthma, reducing
OVA-induced bronchial airway inflammation, cell infiltration and mucin production.

Effect of hPMSC transplantation on cytokine levels in rats with asthma. Serum cytokines were measured by ELISA. A high IgE level indicates a recent allergic attack. The IgE concentrations were significantly higher in the OVA group compared with the control and OVA + hPMSCs groups. The injection of hPMSCs was associated with a significant decrease in IgE in rats challenged with OVA (Fig. 2B). The IL-4 level was higher in the OVA group compared with the control group, although the IFN- $\gamma$ level was lower. In the OVA + hPMSCs group, IL-4 was decreased and IFN- $\gamma$ was increased compared with rats in the OVA group (Fig. 2C and $\mathrm{D} ; \mathrm{P}<0.05)$. The results indicated that hPMSCs inhibited Th2 cytokine secretion and IgE production in rats with OVA-induced asthma.

hPMSC administration alters Notch signaling in lung tissue. The involvement of Notch signaling in asthma and the effects of hPMSCs on Notch signaling were investigated via assessment of Notch-1, Notch-2, Notch-3, delta-4 and jagged-1 expression in lung tissue (Fig. 4). The gene expression of Notch-1, Notch-2 and jagged-1 was significantly higher in the OVA group compared with the control group $(\mathrm{P}<0.05)$; however, it was significantly lower in the OVA + hPMSCs group compared with the OVA group (Fig. 4A, B and D; $\mathrm{P}<0.05)$. Notch-3 and delta-4 expression exhibited the opposite trend, with significantly decreased expression in the OVA group compared with the control and OVA + hPMSCs groups (Fig. $4 \mathrm{C}$ and $\mathrm{E} ; \mathrm{P}<0.05$ ). hPMSC transplantation thus increased Notch-3 and delta-4 expression in the lung tissues of rats treated with OVA + hPMSCs. No significant differences were observed in the expression of Notch receptors and ligands in the control and OVA + hPMSCs groups (Fig. 4C and E). In order to investigate the effects of hPMSCs on Notch signaling responses, protein expression in lung tissue was assayed by western blotting. Consistent with the qPCR results, Notch-1, Notch-2 and jagged-1 protein expression increased in the OVA group compared with the control group $(\mathrm{P}<0.05)$; Notch-3 and delta-4 expression was reduced. The results demonstrated that the administration of hPMSCs decreased the expression of Notch-1, Notch-2, and Jagged-1 and increased Notch-3 and Delta-4 protein expression compared with the expression in the OVA group (Fig. 5). Collectively, the results demonstrated that the Notch pathway had an important role in the lung pathogenesis of asthma and that hPMSCs exerted various effects on Notch receptors and ligands. 

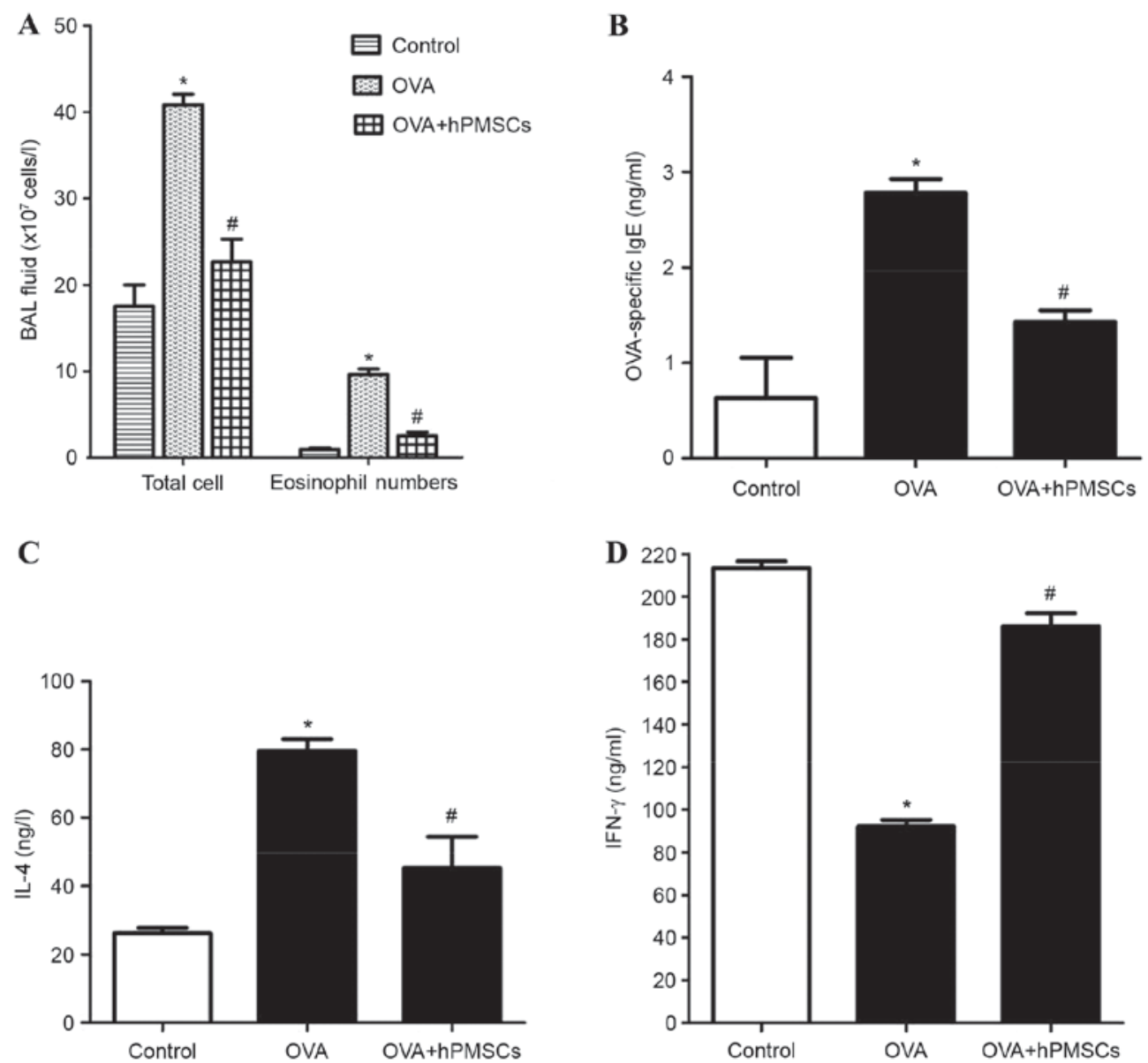

Figure 2. hPMSCs inhibit inflammatory cell infiltration and allergic airway inflammation. (A) Total cells and eosinophils in BAL fluid. Data are presented as percentages of the total cells counted. $\mathrm{n}=10$ /group. (B) Total and OVA-specific serum IgE, analyzed by ELISA. (C) Serum IL-4, analyzed by ELISA. (D) Serum IFN $-\gamma$, analyzed by ELISA. " $\mathrm{P}<0.05$ vs. control group; ${ }^{~} \mathrm{P}<0.05$ vs. OVA group. $\mathrm{n}=10$ /group. BAL, bronchoalveolar lavage; OVA, ovalbumin; IgE, immunoglobulin E; IL-4, interleukin-4; IFN- $\gamma$, interferon- $\gamma$; hPMSCs, human placenta mesenchymal stem cells.

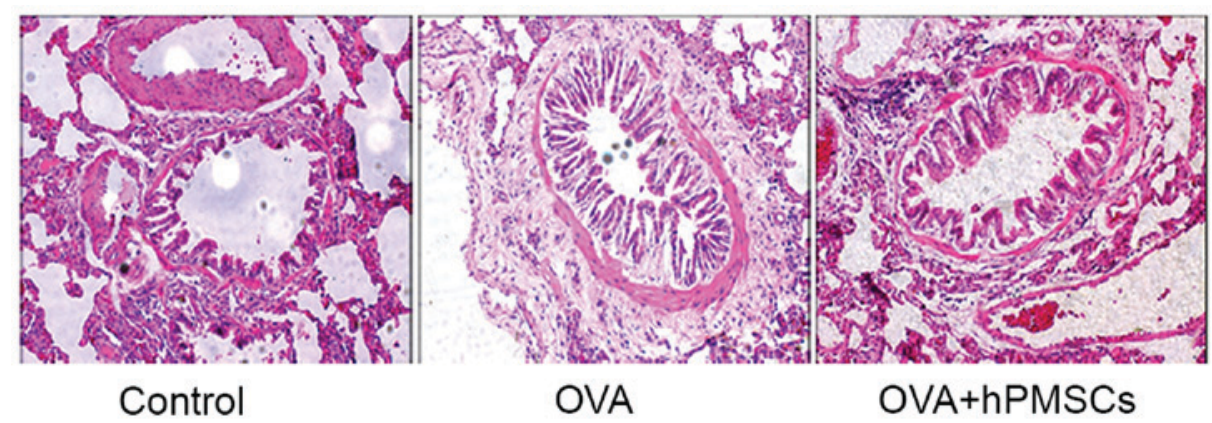

Figure 3. Representative photomicrographs exhibiting histological evidence of inflammation for the asthma model for each experimental condition. Original magnification, x200. Hematoxylin and eosin staining revealed improved lung histopathology in asthmatic rats treated with hPMSCs. OVA, ovalbumin; hPMSCs, human placenta mesenchymal stem cells.

Effect of hPMSCs on Notch signal expression in peripheral blood and lymph. In order to examine the effects of hPMSCs on Notch signaling, Notch-1, Notch-2, Notch-3, delta-4 and jagged-1 gene expression was assayed in peripheral blood and lymph (Fig. 6). Notch-1, Notch-2 and jagged-1 expression was significantly increased in the OVA group compared with the control group $(\mathrm{P}<0.05)$; hPMSC administration significantly increased Notch-1, Notch-2 and jagged-1 expression in the OVA + hPMSCs group ( $\mathrm{P}<0.05$; Fig. 6A, B and D). A similar trend was observed in lymph from the OVA group, with significantly increased Notch-1, Notch-2 and jagged-1 expression compared with the control and OVA + hPMSCs group $(\mathrm{P}<0.05)$. Notch-1, Notch-2 and jagged-1 gene expression was significantly reduced in the OVA + hPMSCs group compared with the OVA group. The results demonstrated that Notch-3 and delta-4 expression in blood and lymph was decreased in the OVA group compared with the control group $(\mathrm{P}<0.05$; Fig. $6 \mathrm{C}$ and $\mathrm{E})$, and was increased by hPMSC transplantation $(\mathrm{P}<0.05)$.

In the OVA group, Notch-1, Notch-2 and jagged-1 gene expression was increased in the lymph compared with the 

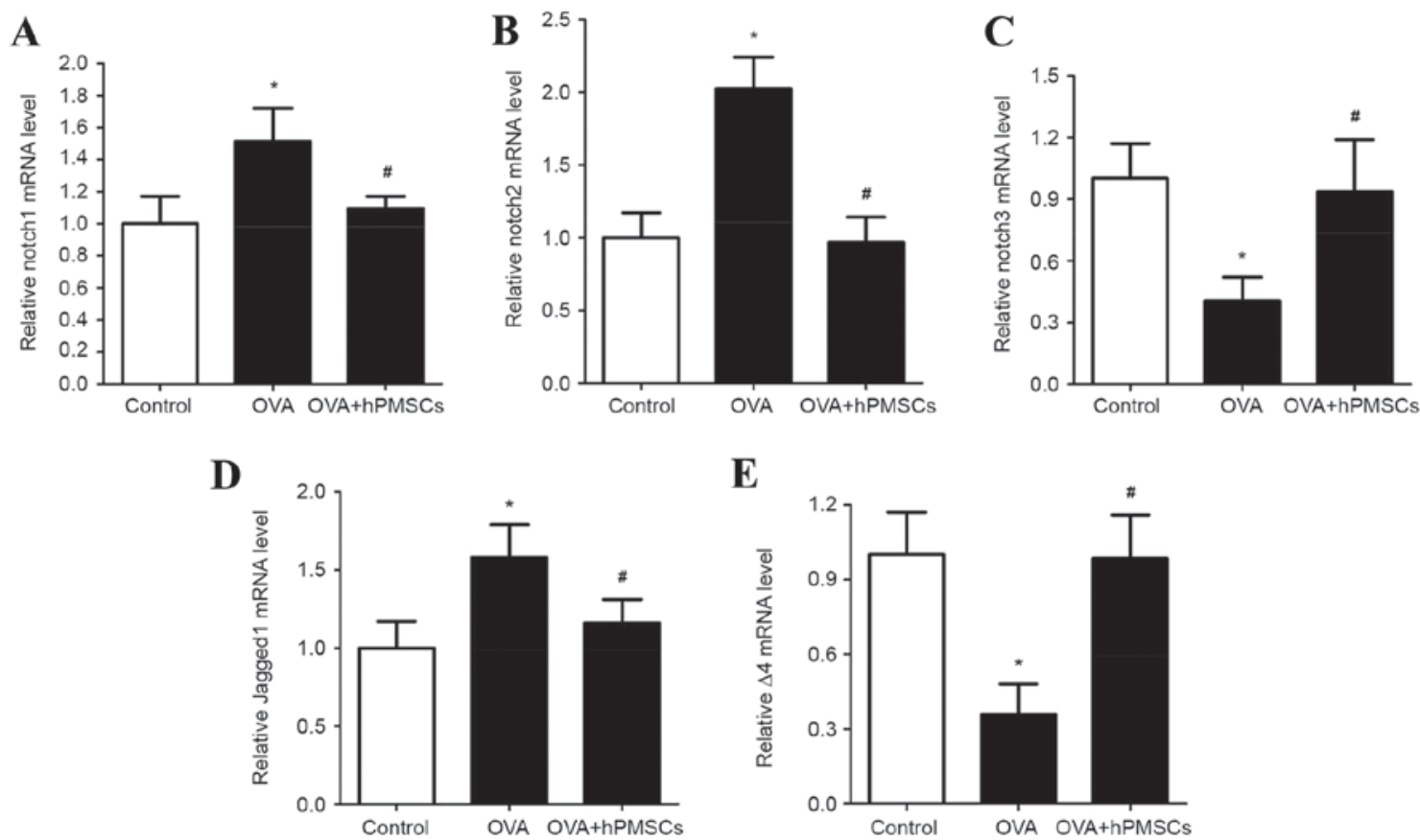

Figure 4. hPMSC engraftment alters the gene expression levels of Notch proteins and ligands in peripheral blood and lymph samples. Notch and ligand expression was assessed by quantitative polymerase chain reaction analysis of lung tissue of control, OVA group and OVA + hPMSCs group rats. (A) Notch-1; (B) Notch-2; (C) Notch-3; (D) jagged-1; (E) $\Delta 4$. "P<0.05 vs. control group; " $\mathrm{P}<0.05$ vs. OVA group. $\mathrm{n}=10$ /group. OVA, ovalbumin; hPMSCs, human placenta mesenchymal stem cells; Notch, neurogenic locus notch homolog protein; $\Delta 4$, delta-like ligand 4.

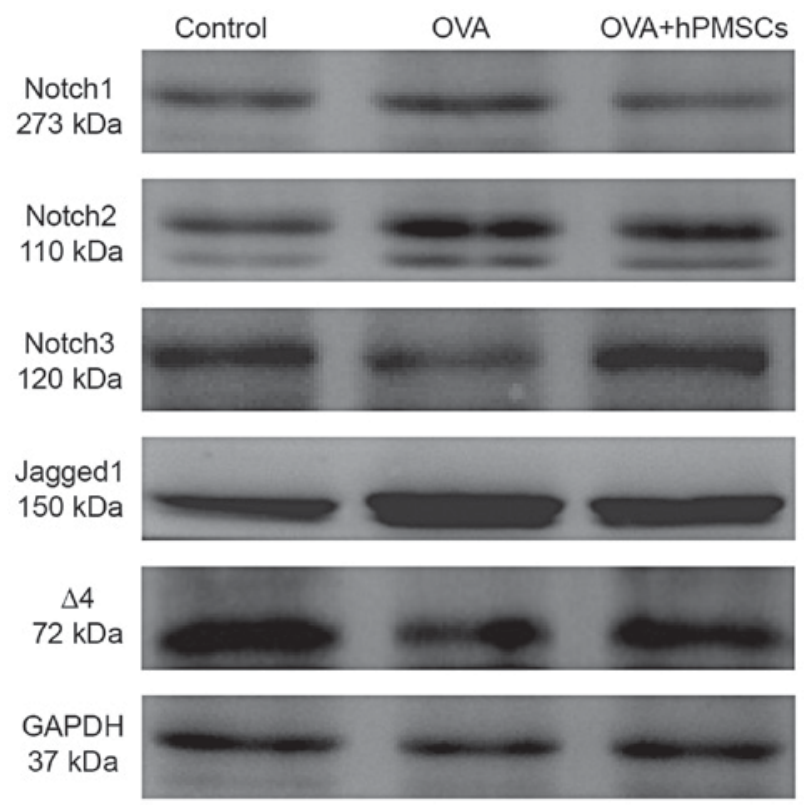

Figure 5. Effects of hPMSC administration on the protein and gene expression levels of Notch proteins and ligands in the lungs of asthmatic rats. Western blot analysis of Notch and ligand protein expression exhibited decreases in Notch-1, Notch-2 and jagged-1 in OVA + hPMSCs rats compared with OVA-induced rats. Notch-3 and $\Delta 4$ expression was increased compared with OVA-induced rats. $n=10 /$ group. OVA, ovalbumin; hPMSCs, human placenta mesenchymal stem cells; Notch, neurogenic locus notch homolog protein; $\Delta 4$, delta-like ligand 4 .

blood $(\mathrm{P}<0.05)$. Meanwhile, Notch-3 and delta-4 expression was decreased $(\mathrm{P}<0.05$; Fig. 6$)$. The results confirmed a greater imbalance in Notch signaling in the lymph compared with the blood, which is consistent with a severe imbalance in T cells, and Notch receptor and ligand expression in the present asthma model.

\section{Discussion}

The present study demonstrated that hPMSCs modulated a regulatory environment in a rat model of asthma via the Notch signaling pathway. Previous studies demonstrated that MSCs have important therapeutic potential in a number of clinical disorders, including asthma (7). Placental-MSCs are similar to bone marrow-derived MSCs in morphology and function, although they exert stronger immune inhibitory effects on T cells $(20,21)$.

A number of studies have used transplanted human MSCs to treat different diseases, and have used them in a variety of animal models, including mice (22), rats (23) and rabbits (24). The findings from these previous studies and those of the present study demonstrate that human MSCs are able to survive and function following transplantation. Yun et al (22) and Chan et al (25) used the method of tail vein injection to transplant MSCs into animals. MSCs may be distributed to the brain and lung through the blood circulation, and the effect of treatment is the same as that when transplanted directly into the location of the lesion. The majority of studies have demonstrated that the primary function of MSCs is an immunosuppressive function, which regulates the immune balance.

The results of the present study demonstrated that hPMSCs decreased the number of lung inflammatory cells and the $\operatorname{IgE}$ serum concentration in OVA-sensitized rats. A significant reduction in the number of eosinophils in the BALF and decreased serum IL-4 added to the evidence that hPMSCs alleviated the asthmatic symptoms and suppressed airway inflammation. The Notch signaling pathway is evolutionarily 

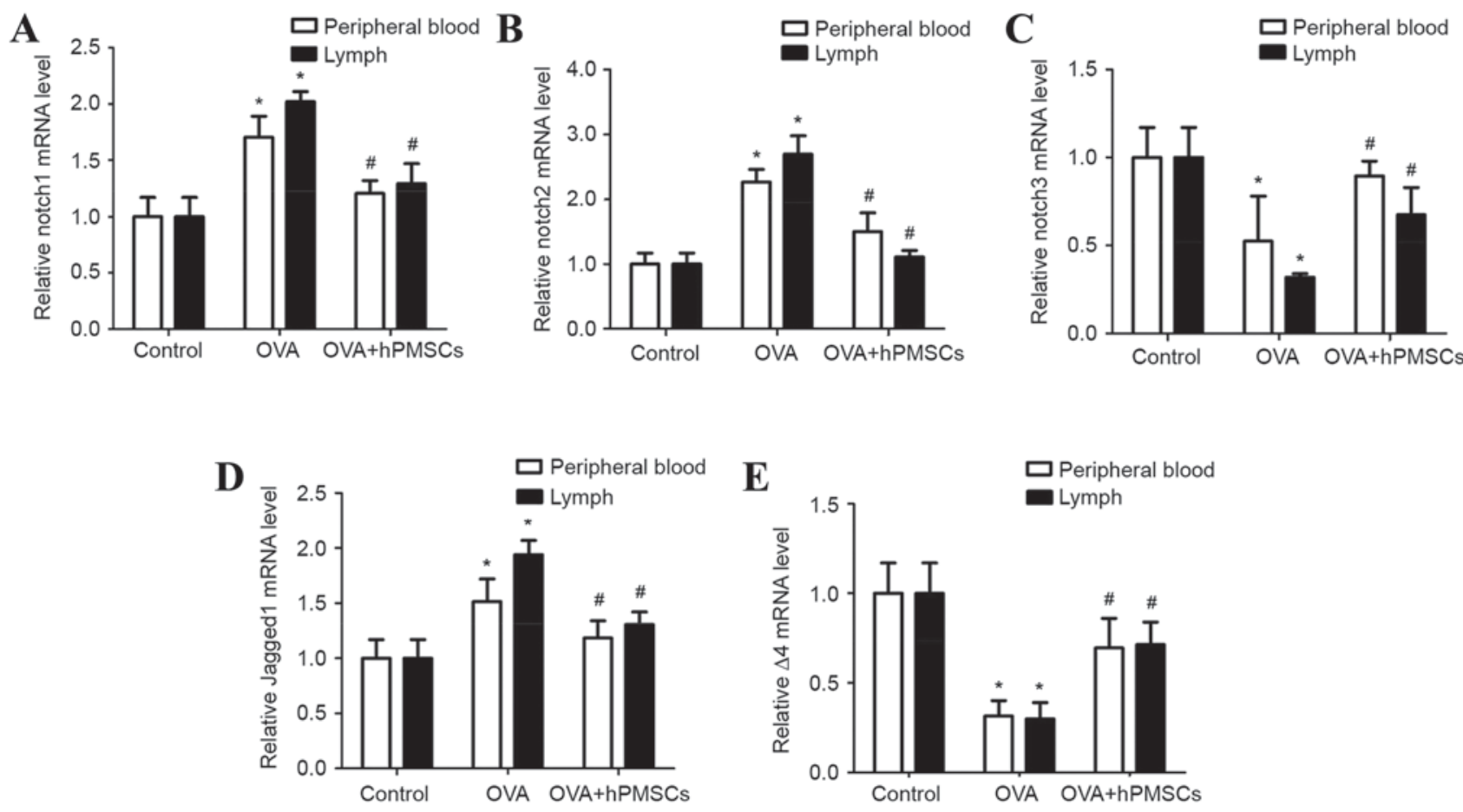

Figure 6. Notch and ligand expression in the lymph and peripheral blood of control, OVA and OVA + hPMSCs group rats. (A) Notch-1; (B) Notch-2; (C) Notch-3; (D) jagged-1; (E) $\Delta 4$. hPMSC-treated rats exhibited significant decreases in Notch-1, Notch-2 and jagged-1 expression in the lymph compared with OVA-induced rats. Notch-3 and $\Delta 4$ expression was increased in hPMSC-treated rats vs. OVA-induced rats. Data are presented as the mean \pm standard deviation. "P<0.05 vs. control group; ${ }^{\text {P }}<0.05$ vs. OVA group. $\mathrm{n}=10$ /group. OVA, ovalbumin; hPMSCs, human placenta mesenchymal stem cells; Notch, neurogenic locus notch homolog protein; $\Delta 4$, delta-like ligand 4.

conserved, and interactions between Notch receptors and ligands regulate cellular differentiation, proliferation and apoptosis. The Notch pathway is involved in a number of diseases, including asthma (26). The Notch pathway has been associated with T-cell development, differentiation and activation, and serves a key role in the induction of $\mathrm{Th} 2$ differentiation $(27,28)$.

Notch expression was observed in the lung tissue and lung $\mathrm{T}$ cells in a BALB/c mouse model of asthma, in which Th2 polarization and Th1/Th2 disturbances in asthma pathogenesis were associated with the Notch pathway (29-31). Guo et al (32) demonstrated Notch-1 expression in lungs and lung $\mathrm{T}$ cells of $\mathrm{BALB} / \mathrm{C}$ mice. The introduction of an activated allele of Notch-1 into $\mathrm{CD} 4^{+} \mathrm{T}$ cells has been demonstrated to activate IL-4 expression and Th2 cell responses (33). In the present study, significant increases were observed in Notch-1 and Notch-2 expression in the lung tissue, blood and lymph of rats with OVA-induced asthma, and serum IL-4 mRNA was increased. Following hPMSC administration, Notch-1 and Notch-2 expression decreased and IFN- $\gamma$ increased. Previous studies have demonstrated that Notch-1 and Notch-2 interact with jagged-1/2 to induce Th2-cell differentiation following treatment with Th2-cell stimuli (34-36). Notch-1 and Notch-2 were analyzed in a mouse asthma model, and the effect of Notch-1 was stronger compared with that of Notch-2 (37).

In the present study, a difference was not observed between Notch-1 and Notch-2 expression. The results confirmed a role for Notch signaling in asthma pathogenesis, and that hPMSCs may inhibit Th2 polarization by blocking Notch signaling, which may attenuate the excessive production of
Th2 cytokines. Previous reports have suggested that CD4 ${ }^{+}$ T-cell subsets, including Th17 cells and Treg cells, may serve key roles in asthma (38-41). Notch-3 signaling and cell-to-cell contact facilitates Treg-cell expansion. MSCs were demonstrated to decrease cell infiltration and lung pathology via regulation of the Treg-cell population in a previous model of allergic asthma (17). In the present study, Notch-3 expression was observed in the lung tissue, blood and lymph in all three study groups.

Previous research demonstrated that the percentages of $\mathrm{CD} 4^{+} \mathrm{CD} 25^{+}$Foxp $^{+}$Tregs were significantly decreased in the serum of OVA group rats compared with those in the control group and hPMSC-treated rats. The administration of hPMSCs markedly increased the percentages of $\mathrm{CD} 4{ }^{+} \mathrm{CD} 25^{+}$ Foxp $^{+}$Tregs in the serum and lymph. The Th17/Treg rebalance was induced by hPMSC administration. The mechanism may be mediated by Treg regulation, partly involving increased IL-10 levels and Foxp3/ROR $\gamma t$ (42). These previous results suggested that Notch-3 signaling may be involved in the development of asthma by regulating the generation and the expansion of Treg cells through hPMSC administration. Notch receptors and ligands are involved in the regulation of immune cells and tissue microenvironments (26). In the present study, jagged-1 and delta-4 mRNA were detected in the lungs, serum and lymph of the control group, OVA group and hPMSC-treated group, and Jagged-1 expression was increased in the rats with OVA-induced asthma. Treatment with hPMSCs decreased the jagged-1 response to OVA challenge. Delta- 4 expression decreased in the rats with OVA-induced asthma compared with the control group 
and the OVA+hPMSCs group. Jagged-1 was required for the expansion of $\mathrm{CD} 4{ }^{+} \mathrm{CD} 25^{+}$FoxP $3{ }^{+}$regulatory $\mathrm{T}$ cells following treatment with MSCs (43). Rutz et al (3) demonstrated that delta-1 and jagged-1 served important roles in the inhibition of T-cell activation, whereas delta-4 expression enhanced T-cell proliferation. These results provide an insight into the involvement of Notch signaling in the crosstalk between immune cells and hPMSCs in asthma.

In conclusion, the results of the present study demonstrated that hPMSCs may reduce airway inflammation via inhibition of Notch-1, Notch-2 and jagged-1 expression, and promotion of Notch-3 and delta-4 expression. The effect of hPMSC administration in alleviating airway hyper-responsiveness and inflammation may be mediated by Notch regulation, partly involving reduced IL-4 and IgE levels and increased IFN- $\gamma$. hPMSCs may be a feasible agent for treating asthma in the future. The present findings add to the understanding of the biological significance of hPMSCs in mediating the Notch pathway in asthma pathogenesis.

\section{Acknowledgements}

The present study was supported by grants from the Shandong Province Natural Science Foundation of China (grant no. ZR2011HM081), Yantai Science and Technology Development Funds (grant no. 2008142-11) and the Taishan Scholar Foundation.

\section{References}

1. Kupczyk M and Wenzel S: U.S. and European severe asthma cohorts: What can they teach us about severe asthma? J Intern Med 272: 121-132, 2012.

2. Wenzel S: Severe asthma: From characteristics to phenotypes to endotypes. Clin Exp Allergy 42: 650-658, 2012.

3. Rutz S, Mordmüller B, Sankano S and Scheffold A: Notch ligands Delta-like1, Delta-like4 and Jagged1 differentially regulate activation of peripheral $\mathrm{T}$ helper cells. Eur J Immunol 35: 2443-2451, 2005.

4. Rao PE, Petrone AL and Ponath PD: Differentiation and expansion of $\mathrm{T}$ cells with regulatory function from human peripheral lymphocytes by stimulation in the presence of TGF-\{beta\}. J Immunol 174: 1446-1455, 2005.

5. Del Papa B, Sportoletti P, Cecchini D, Rosati E, Balucani C, Baldoni S, Fettucciari K, Marconi P, Martelli MF, Falzetti F and Di Ianni M: Notch1 modulates mesenchymal stem cells mediated regulatory T-cell induction. Eur J Immunol 43: 182-187, 2013.

6. Bassi ÊJ, Moraes-Vieira PM, Moreira-Sá CS, Almeida DC, Vieira LM, Cunha CS, Hiyane MI, Basso AS, Pacheco-Silva A and Câmara NO: Immune regulatory properties of allogeneic adipose-derived mesenchymal stem cells in the treatment of experimental autoimmune diabetes. Diabetes 61: 2534-2545, 2012.

7. Mariñas-Pardo L, Mirones I, Amor-Carro O, Fraga-Iriso R, Lema-Costa B, Cubillo I, Rodríguez Milla MÁ, García-Castro J and Ramos-Barbón D: Mesenchymal stem cells regulate airway contractile tissue remodeling in murine experimental asthma. Allergy 69: 730-740, 2014.

8. Bassi EJ, Aita CA and Câmara NO: Immune regulatory properties of multipotent mesenchymal stromal cells: Where do we stand? World J Stem Cells 3: 1-8, 2011.

9. Machado Cde V, Telles PD and Nascimento IL: Immunological charac-teristics of mesenchymal stem cells. Rev Bras Hematol Hemoter 35: 62-67, 2013.

10. Aggarwal S and Pittenger MF: Human mesenchymal stem cells modulate allogeneic immune cell responses. Blood 105: 1815-1822, 2005.

11. Asari S, Itakura S, Ferreri K, Liu CP, Kuroda Y, Kandeel F and Mullen Y: Mesenchymal stem cells suppress B-cell terminal differentiation. Exp Hematol 37: 604-615, 2009.
12. Ryan JM, Barry FP, Murphy JM and Mahon BP: Mesenchymal stem cells avoid allogeneic rejection. J Inflamm (Lond) 2: 8, 2005.

13. Jonuleit H, Schmitt E, Schuler G, Knop J and Enk AH: Induction of interleukin 10-producing, nonproliferating CD4(+) T cells with regulatory properties by repetitive stimulation with allogeneic immature human dendritic cells. J Exp Med 192: 1213-1222, 2000.

14. Yamazaki S, Iyoda T, Tarbell K, Olson K, Velinzon K, Inaba K and Steinman RM: Direct expansion of functional CD25+ CD4+ regulatory $\mathrm{T}$ cells by antigen-processing dendritic cells. J Exp Med 198: 235-247, 2003.

15. Morelli AE and Thomson AW: Tolerogenic dendritic cells and the quest for transplant tolerance. Nat Rev Immunol 7: 610-621, 2007.

16. Maldonado RA and von Andrian UH: How tolerogenic dendritic cells induce regulatory T cells. Adv Immunol 108: 111-165, 2010

17. Kavanagh $\mathrm{H}$ and Mahon BP: Allogeneic mesenchymal stem cells prevent allergic airway inflammation by inducing murine regulatory T cells. Allergy 66: 523-531, 2011.

18. Yang M, Zhao X, Liu Y, Tian Y, Ran X and Jiang Y: A role for WNT1-inducible signaling protein-1 in airway remodeling in a rat asthma model. Int Immunopharmacol 17: 350-357, 2013.

19. Livak KJ and Schmittgen TD: Analysis of relative gene expression data using real-time quantitative PCR and the 2(-Delta Delta C(T)) method. Methods 25: 402-408, 2001.

20. Srour N and Thébaud B: Stem cells in animal asthma models: A systematic review. Cytotherapy 16: 1629-1642, 2014.

21. Li X, Bai J, Ji X, Li R, Xuan Y and Wang Y: Comprehensive characterization of four different populations of human mesenchymal stem cells as regards their immune properties, proliferation and differentiation. Int J Mol Med 34: 695-704, 2014.

22. Yun HM, Kim HS, Park KR, Shin JM, Kang AR, il Lee K, Song S, Kim YB, Han SB, Chung HM and Hong JT: Placenta-derived mesenchymal stem cells improve memory dysfunction in an A $\beta 1-42$-infused mouse model of Alzheimer's disease. Cell Death Dis 4: e958, 2013.

23. Kong P, Xie X, Li F, Liu Y and Lu Y: Placenta mesenchymal stem cell accelerates wound healing by enhancing angiogenesis in diabetic Goto-Kakizaki (GK) rats. Biochem Biophys Res Commun 438: 410-419, 2013.

24. Stoff A, Rivera AA, Sanjib Banerjee N, Moore ST, Michael Numnum T, Espinosa-de-Los-Monteros A, Richter DF, Siegal GP, Chow LT, Feldman D, et al: Promotion of incisional wound repair by human mesenchymal stem cell transplantation. Exp Dermatol 18: 362-369, 2009.

25. Chan CK, Lin TC, Huang YA, Chen YS, Wu CL, Lo HY, Kuo ML, Wu KH and Huang JL: The modulation of Th2 immune pathway in the immunosuppressive effect of human umbilical cord mesenchymal stem cells in a murine asthmatic model. Inflamm Res 65: 795-801, 2016

26. Radtke F, Wilson A, Mancini SJ and MacDonald HR: Notch regulation of lymphocyte development and function. Nat Immunol 5: 247-253, 2004.

27. Krishnaswamy S, Verdile G, Groth D, Kanyenda L and Martins RN: The structure and function of Alzheimer's gamma secretase enzyme complex. Crit Rev Clin Lab Sci 46: 282-301, 2009.

28. Berezovska O, Jack C, Deng A, Gastineau N, Rebeck GW and Hyman BT: Notch1 and amyloid precursor protein are competitive substrates for presenilin1-dependent gamma-secretase cleavage. J Biol Chem 276: 30018-30023, 2001.

29. Cui ZL, Gu W, Ding T, Peng XH, Chen X, Luan CY, Han RC, $\mathrm{Xu}$ WG and Guo XJ: Histone modifications of Notch1 promoter affect lung CD4+ T cells differentiation in asthmatic rats. Int $\mathrm{J}$ Immunopathol Pharmacol 26: 371-381, 2013.

30. De Strooper B, Annaert W, Cupers P, Saftig P, Craessaerts K, Mumm JS, Schroeter EH, Schrijvers V, Wolfe MS, Ray WJ, et al: A presenilin-1-dependent gamma-secretase-like protease mediates release of Notch intracellular domain. Nature 398: 518-522, 1999.

31. Karlström H, Bergman A, Lendahl U, Näslund J and Lundkvist J: A sensitive and quantitative assay for measuring cleavage of presenilin substrates. J Biol Chem 277: 6763-6766, 2002.

32. Guo XJ, Zhou M, Ren LP, Yang M, Huang SG and Xu WG: Small interfering RNA mediated knockdown of Notch1 in lung T cells of asthmatic mice affects $\mathrm{T}$ cell differentiation. Chin Med J (Engl) 122: 2647-2651, 2009.

33. Fang TC, Yashiro-Ohtani Y, Del Bianco C, Knoblock DM, Blacklow SC and Pear WS: Notch directly regulates Gata3 expression during $\mathrm{T}$ helper 2 cell differentiation. Immunity 27 : 100-110, 2007. 
34. Osborne BA and Minter LM: Notch signalling during peripheral T-cell activation and differentiation. Nat Rev Immunol 7: 64-75, 2007.

35. Bailis W, Yashiro-Ohtani Y, Fang TC, Hatton RD, Weaver CT, Artis D and Pear WS: Notch simultaneously orchestrates multiple helper $\mathrm{T}$ cell programs independently of cytokine signals. Immunity 39: 148-159, 2013.

36. Okamoto M, Matsuda H, Joetham A, Lucas JJ, Domenico J, Yasutomo K, Takeda K and Gelfand EW: Jagged1 on dendritic cells and Notch on CD4+ T cells initiate lung allergic responsiveness by inducing IL-4 production. J Immunol 183: 2995-3003, 2009.

37. Zong D, Ouyang R, Li J, Chen Y and Chen P: Notch signaling in lung diseases: Focus on Notch1 and Notch3. Ther Adv Respir Dis 10: 468-484, 2016.

38. Shi YH, Shi GC, Wan HY, Jiang LH, Ai XY, Zhu HX, Tang W, Ma JY, Jin XY and Zhang BY: Coexistence of Th1/Th2 and Th17/Treg imbalances in patients with allergic asthma. Chin Med J (Engl) 124: 1951-1956, 2011.

39. Xu W, Lan Q, Chen M, Chen H, Zhu N, Zhou X, Wang J, Fan H, Yan CS, Kuang JL, et al: Adoptive transfer of induced-Treg cells effectively attenuates murine airway allergic inflammation. PLoS One 7: e40314, 2012.
40. Kearley J, Robinson DS and Lloyd CM: CD4+CD25+ regulatory $\mathrm{T}$ cells reverse established allergic airway inflammation and prevent airway remodeling. J Allergy Clin Immunol 122: 617-624.e6, 2008.

41. Kared H, Adle-Biassette H, Foïs E, Masson A, Bach JF, Chatenoud L, Schneider E and Zavala F: Jagged2-expressing hematopoietic progenitors promote regulatory $\mathrm{T}$ cell expansion in the periphery through notch signaling. Immunity 25: 823-834, 2006.

42. Li Y,LiH,Cao Y,Wu F,MaW,Wang Y and Sun S: Placenta-derived mesenchymal stem cells improve airway hyperresponsiveness and inflammation in asthmatic rats by modulating the Th17/Treg balance. Mol Med Rep 16: 8137-8145, 2017.

43. Cahill EF, Tobin LM, Carty F, Mahon BP and English K: Jagged-1 is required for the expansion of CD4+CD25+ FoxP3+ regulatory $\mathrm{T}$ cells and tolerogenic dendritic cells by murine mesenchymal stromal cells. Stem Cell Res Ther 6: 19, 2015. 